\title{
COMPETENCIAS PARA LA EDUCACIÓN SUPERIOR EN EL ECUADOR DEL SIGLO XXI: RESPONSABILIDAD SOCIAL Y TECNOLOGÍAS
}

\author{
COMPETENCES FOR HIGHER EDUCATION IN ECUADOR \\ OF THE XXI CENTURY: SOCIAL RESPONSIBILITY \\ AND TECHNOLOGIES
}

\author{
Rita P. Pérez Zamora', Eugenia del C. Miño Acurio², \\ María C. Miño Acurio ${ }^{3}$ Wilma M. Feijoó Vega
}

\section{RESUMEN}

El presente artículo aborda la responsabilidad social de las Instituciones de Educación en Ecuador para formar en competencias que inciden en el respeto y reconocimiento de la riqueza en diversidad cultural y de biodiversidad, y por otra parte en competencias digitales para preparar a estudiantes ante la revolución tecnológica a nivel global. El objetivo es aportar a investigadores y docentes del Ecuador un aporte desde la literatura internacional para tener referentes para aplicar en el contexto de su trabajo académico. La investigación es de carácter descriptivo, no experimental fundamentado en revisión de bibliográfica y documentación. Los resultados que se presentan constatan los avances conseguidos en el país desde la constitución de Monte Cristi en materia de atribuir una responsabilidad social a la educación y universalizarla gratuitamente a toda la población al tiempos que disponiendo medidas para impulsar su calidad; de igual modo se señala como debe realizarse un mayor trabajo en proporcionar metodologías para la implementación de las tecnologías y capacitación docente en su uso pedagógico.

1 Magister en diseño curricular y evaluación educativa, Docente en Colegio Particular San Pio X, Huachi Grande Barrio “La Palestina”, Ambato, Ecuador. rpperez@uta.edu.ec

2 Licenciada en ciencias de la educación. Docente en Unidad Educativa "Leonardo Murialdo".

3 Licenciada en ciencias de la educación. Asesora en la Asamblea Nacional de Ecuador.

4 Magister en diseño curricular y evaluación educativa. Docente en Universidad Técnica de Ambato. 
Se concluye que la situación de la Educación Superior en Ecuador ha conseguido notables avances en la última década, subrayando la importancia de continuar en el fortalecimiento de todo el ciclo educativo para que al llegar a la universidad los estudiantes puedan aprovechar al máximo los recursos a su disposición y contribuir al desarrollo de cada una de las regiones y así al país.

PALABRAS CLAVE: Ecuador, competencias, tecnologías información y comunicación, responsabilidad social. Sumak Kawsay.

\section{ABSTRACT}

This article deals with the social responsibility of the Educational Institutions in Ecuador to train in competitions that affect the respect and recognition of the richness in cultural diversity and biodiversity, and on the other hand in digital competences to prepare students before the technological revolution At the global level. The objective is to provide researchers and teachers of Ecuador with a contribution from the international literature to have references to apply in the context of their academic work. The design of the research is descriptive, not experimental, based on bibliographical review and documentation. Results confirm the progression achieved in the country since the constitution of Monte Cristi in the attribution of a social responsibility to education and universalize it free of charge to the entire population at times that providing measures to boost its quality. It also indicates how more work should be done in providing methodologies for the implementation of information and communication technologies, also teacher training in its pedagogical use. It is concluded that the situation of Higher Education in Ecuador has achieved remarkable progress in the last decade, underlining the importance of continuing to strengthen the entire educational cycle so that students upon arrival at the university can make the most of the resources at their disposal and thus contribute to the development of each of the regions and thus the country.

KEY WORDS: Ecuador, competences, information and communication technologies, social responsibility, Sumak Kawsay.

\section{INTRODUCCIÓN}

El artículo centra su atención en la Responsabilidad Social de la Educación que en el marco de la transformación que imprime la Constitución del Ecuador conocida como la Constitución de Montecristi (Asamblea Nacional Constituyente, 2008), y que tiene como misión garantizar que las personas, comunidades, pueblos y nacionalidades gocen de sus derechos el marco de la interculturalidad, del respeto a su diversidad en convivencia armónica con la naturaleza acometiendo un cambio en el modelo socioeconómico del país en el que la Educación, y en especial la universitaria, adquiere un papel fundamental de gran trascenden- 
cia para formar a estudiantes para una ciudadanía culta, preparada y socialmente responsable.

Para esta finalidad, resulta prioritario el comprender qué competencias desde la educación deben desarrollarse y fortalecerse en estudiantes desde las potencialidades del país por una parte; mientras que por otra parte atendiendo al contexto de mundo globalizado en el cual estamos inmersos conviene estar atentos a las políticas y tendencias educativas (Rafael, 2015), y actualmente ya se refiere a una IV revolución industrial que se caracteriza por una preponderancia de las tecnologías de la información y comunicación y va a requerir nuevas competencias para trabajar (World Economic Forum, 2015).

Por lo tanto, este artículo de investigación toma en cuenta esta doble confluencia en torno a la educación, la requerida para impulsar el desarrollo humano del Ecuador en función de la riqueza cultural y biodiversidad, así como las competencias tecnológicas que deben adquirirse a lo largo de la formación para estar integrados en las dinámicas de la globalización. A fin de realizar un aporte significativo, se comenta bibliografía y documentos latinoamericanos e internacionales para facilitar a pedagogos, docentes, administradores de política pública y la población en general, un porte a tener presente para formar a estudiantes desde un enfoque humanista.

\section{MÉTODOS}

La investigación corresponde a un estudio descriptivo no experimental, que se fundamenta en una revisión de bibliográfica y documental en torno a las dos variables mencionadas: competencias para una ciudadanía socialmente responsable y competencias digitales. Si bien, el aprendizaje implícito que se da durante el proceso investigador, el trabajo va más allá del objetivo inicial realizando algunos apuntes complementarios a las necesidades de las instituciones educativas y de la formación en el siglo XXI.

En primer lugar se presenta una argumentación sobre competencias para la responsabilidad social, mientras que seguidamente se aborda el proceso de enseñanza y aprendizaje de las tecnologías aplicadas a la formación en la cual se pone en relieve que si bien muchos programas se han orientado a dotar de infraestructura, la realidad que se impone es la de un perfil de estudiante que se familiariza con más rapidez que el docente en su uso y manejo generando una relación simétrica de acceso a conocimiento. 


\section{COMPETENCIAS Y EDUCACIÓN SOCIALMENTE RESPONSABLE}

Ecuador mediante un seguido de políticas públicas se comprometió con la educación y cada estudiante a lograr el éxito y el bienestar. El principal objetivo del sistema educativo es desarrollar los conocimientos, habilidades y competencias para que quienes estudian se vean enriquecidos en lo personal, y en la trayectoria que les conduzca hacia la educación superior, y se profesionalicen, devengan económicamente productivos y ciudadanos comprometidos (Asamblea Nacional República del Ecuador, 2010; Asamblea Nacional República del Ecuador, 2016).

La madurez en el crecimiento intelectual no requiere antiguos sistemas de memorizar contenidos, sino que se reconoce la necesidad de promover el pensamiento crítico y creativo para la resolución de problemas y la innovación (Tamayo, Zona \& Loaiza, 2015; Trilling \& Fadel, 2009). Drake (2014) destaca el desafío de diseñar programas educativos con experiencias que abordan los problemas locales y los problemas del mundo real. Puede que no haya una respuesta clara y pensar críticamente requiere que los estudiantes rompan paradigmas previos en los que la educación ha sido una forma de sometimiento al estado de orden del sistema. Sin embargo, en Ecuador los cambios en la educación hacia la integración de las diferentes culturas que lo conforman para que contribuyan con sus conocimientos al desarrollo del país (Ministerio de Educación, 2012), han puesto de relevancia el aprendizaje significativo en el cual resulta básico el situar el foco de la enseñanza en el aprendizaje vía los conocimientos que aporta cada estudiante generando interactividad en el aula y discutiendo las diferentes formas de comprender un mismo concepto (López, Veit y Solano Araujo, 2014; Olaya y Ramírez, 2015).

Por ello, en el siglo XXI la novedad que urge es el llamado a los sistemas educativos para enfatizar y desarrollar estas competencias de manera explícita e intencional a través de cambios deliberados en el diseño de los planes de estudios y la práctica pedagógica. El objetivo pretendido con estos cambios en la educación es formar en competencias para preparar a estudiantes para resolver problemas complejos y desordenados -incluidos los problemas de los que aún no se sabe respuesta- asociados con la vida desde la perspectiva de cada cultura, en un mundo competitivo y globalmente conectado. 
En todo caso, ia qué nos referimos al hablar de competencias? Según el glosario de la Comisión Europea las competencias son definidas como la capacidad de aplicar los resultados de aprendizaje adecuadamente en un entorno personal, de educación o profesional que no se limita a elementos cognitivos (la teoría) sino también aspectos funcionales que implican habilidades técnicas, así como aspectos interpersonales de relación con los demás y valores éticos. Por lo tanto, es un concepto amplio que en realidad comprende habilidades, actitudes, conocimientos, emociones, etc. (Centro Europeo para el Desarrollo de la Formación Profesional, 2014). La educación no solamente debe quedarse en trasladar conocimientos, alejados de la realidad humana y social que los rodea y en función de ello el diálogo establecido en torno a las competencias las sitúa en la esfera más profunda e íntima del ser al relacionarla con los valores y una ética personal que trascienda a lo profesional.

Muchos estudios demuestran la importancia de la creatividad para el desarrollo social, la capacidad de competir en los negocios y la capacidad de generar crecimiento económico, entre ellos los resultados del informe PISA 2012 (OCDE, 2014) señalan la conexión entre alto rendimiento académico, la resolución de problemas y la creatividad; esta situación va acorde al Plan Nacional del Buen Vivir (Secretaría Nacional de Planificación y desarrollo, 2013) en el que se establecen las bases para acometer una transformación de la matriz productiva y pasar de un modelo socio-económico de país exportar de materia prima a una economía que tenga en el conocimiento y la innovación su principal eje de desarrollo con la capacidad humana que alberga Ecuador (Secretaría Nacional de Planificación y desarrollo, 2013).

En cuanto a su conceptualización y organización, a nivel internacional se han destacado una serie de competencias clave que pueden identificarse sobre la base de su contribución al logro educativo, las relaciones, el desarrollo laboral así como en la Salud y el bienestar, y lo hacen para todas las personas, no sólo para aquellas que trabajan en determinadas profesiones (Rychen, 2003). Las competencias consideradas clave para el siglo XXI son el Pensamiento Crítico, la Comunicación, la Colaboración y la Creatividad e innovación; seguidamente en la Tabla 1 se presenta una breve definición de qué se entiende por cada una para así acceder a una panorámica de cómo adaptarlas a la esfera educativa del Ecuador. 
Tabla 1

COMPETENCIAS CLAVE

\section{Pensamiento crítico}

"La capacidad de diseñar y gestionar proyectos, resolver problemas y adoptar decisiones eficaces utilizando una variedad de herramientas y recursos" (Fullan, 2013). "Adquirir, procesar, interpretar, racionalizar y analizar críticamente grandes volúmenes de información a menudo conflictiva hasta el punto de llegar a la decisión informada y actuación oportuna" (C21, 2012: 10).

\section{Comunicación}

"La comunicación en un contexto siglo 21 se refiere no sólo a la capacidad de comunicarse de manera efectiva, oral, por escrito, y con una variedad de herramientas digitales, sino también a habilidades de escucha" (Fullan, 2013, p. 9).

\section{Colaboración}

Requiere la capacidad de "trabajar en equipo, aprender y contribuir al aprendizaje de otros, [utilizar] las habilidades de redes sociales, [y demostrar] la empatía trabajando con diversos otros" (Fullan, 2013, p.9). Requiere que los estudiantes desarrollen inteligencia colectiva y coconstruyan, lo que significa, convertirse en creadores de contenido y aprender de forma conectada (Siemens, 2005).

\section{Creatividad y la innovación}

La creatividad se describe como la búsqueda de nuevas ideas, conceptos o productos que satisfagan una necesidad mundo. La innovación contiene elementos de creatividad y se describe como la realización de una nueva idea con el fin de hacer una útil contribución a un campo particular. La creatividad incluye conceptos de "economía y emprendimiento social y liderazgo para la acción” (Fullan, 2013: 9).

Fuente: Autor. 
La responsabilidad social en la educación es un tema muy relevante para la realidad iberoamericana (Martí Noguera, Arango y Moncayo, 2015), internacionalmente aceptado como finalidad de la educación el promover ciudadanía y responsabilidad. Sin embargo tal vez una de las grandes fallas es que no hay un sistema de control que evalúe la adquisición en competencias de responsabilidad social. En Ecuador un primer paso, a nivel de universidades, radica en los procesos de acreditación entre cuyos indicadores si se refiere abiertamente a la responsabilidad social y la ética (Consejo de Evaluación, Acreditación y Aseguramiento de la calidad de la Educación Superior, 2015), cuanto menos empieza a figurar a nivel organizacional y en este artículo se pone de manifiesto que debe ser un tema central en cuanto a la formación y su evaluación.

\section{COMPETENCIAS EN TECNOLOGÍAS}

Indica Dede (2010) que además de colaborar cara a cara con colegas en un mismo espacio, en este siglo XXI la población realiza cada vez más tareas a través de interacciones mediadas por entornos virtuales con sus compañeros situados en diferentes lugares, y que tal vez nunca puedan encontrarse cara a cara. Así, incluso aunque en naturaleza la colaboración es una de las capacidades interpersonales cooperativas más desarrolladas, los nuevos entornos exigen habilidades involucradas son más sofisticadas que en la era anterior dando paso a la necesidad de formar en competencias tecnológicas o también conocidas como digitales.

Melo, Silva Chávez, Indacochea Mendoza y Núñez Campaña (2017) ponen de relieve cómo el proceso de inmersión tecnológica en las instituciones de educación ha sufrido diversos problemas, revelando una baja comprensión del proceso de enseñanza aprendizaje al haber situado algunos gobiernos la prioridad en invertir en infraestructura más que en atender al proceso educativo (Adell \& Castañeda, 2012). Diversos estudios referidos muestran como el conectivismo emerge como una teoría en la que saliendo de paradigmas educativos vigentes, Siemens (2005) pone la atención en espacios virtuales en los que el conocimiento está creándose constantemente en interacción entre personas; esta situación genera una indefensión en docentes que han aprendido a enseñar en contextos de aula atendiendo a unos materiales estáticos. Si bien hay conocimientos elementales, el conectivismo no rebate el hecho de aprender de bases comunes, sino más bien alimenta la oportunidad que supone un acceso desde múltiples puntos de vista lo cual requiere otros enfoques pedagógicos y repensar el aula como espacio de formación (Delgadillo Franco \& Islas Torres, 2016; Sobrino Morrás, 2014; Zapata-Ros, 2015). 
Aunque el caso de Ecuador es de un país con un creciente número de instituciones con acceso a tecnología y conectividad, es necesario atender a experiencias internacionales que enfocan a los cambios que se dan en estudiantes al margen del aprendizaje formal (Organization Economic Cooperation Development, 2015), de la misma manera en que es básico fortalecer los intercambios de aprendizajes con países de la región (Montero Pereda, 2014; Nizama Reyes, 2016).

\section{RESULTADOS}

La Organización para la Cooperación y el Desarrollo Económico (OCDE) instó a los gobiernos internacionales a "hacer un esfuerzo para identificar y conceptualizar el conjunto de competencias y competencias necesarias para incorporar los estándares educativos que cada estudiante debería ser capaz de llegar al final de la escolaridad obligatoria" (Ananiadou \& Claro, 2009, p.5). No obstante, las competencias son multifacéticas y abordadas en múltiples estudios, por lo que entre las limitaciones de este artículo está fuera del alcance de este documento explorar en profundidad su alcance global e influencia en cuanto a la formación para el desarrollo humano y su participación en sociedad.

Sin embargo, se cumple el objetivo señalado de aportar referentes sobre dos aspectos considerados básicos, su alcance en enlazar la educación con el desarrollo de una ciudadanía socialmente responsable y la influencia internacional que las tecnologías han adquirido independientemente del contexto cultural, si bien, como se ha señalado permanecer al margen es una opción que debe considerarse atendiendo al modelo educativo del país basado en el reconocimiento pluricultural.

A modo de resumen, los sistemas de educación para seguir el ritmo de los tiempos cambiantes de este Siglo XXI se encuentra a menudo relacionados con:

$\checkmark$ Cambios en la concepción del desarrollo socio-económico y los modelos de trabajo a partir de un modelo industrial de producción que con los avances se transforma rápidamente, impulsado por la tecnología y una economía del conocimiento interconectada y globalizada. Tal economía requiere desarrollar competencias adaptadas a modelos dinámicos e impredecibles de desarrollo económico y social.

$\checkmark$ Evidencia sobre la emergente manera de optimizar el aprendizaje, incluyendo el uso de innovaciones tecnológicas para profundizar y transformar las relaciones de enseñanza-aprendizaje. 
$\checkmark \quad$ El cambio de expectativas por parte de los estudiantes, que exigen un sistema educativo más conectado y relevante a sus necesidades en las que la tecnología pasa a ser un nuevo medio de interacción y aprendizaje desde lo formal e informal (Benavides \& Pedró, 2007; Claro, 2010; Dumont, Istance y Benavides, 2010).

\section{DISCUSIÓN}

La evolución de la Educación Superior en el mundo, y en América Latina en particular (Adams Becker, Cummins, Davis, Freeman, Hall Giesinger y Ananthanarayanan, 2017; New Media Consortium, 2013) se enfrente a cambios más acelerados de lo que probablemente nunca antes estuvo, mediada por avances tecnológicos y una gran movilidad de gentes en un entorno interconectado el hecho de tener a mayor población con acceso a la educación aun no ha logrado solucionar retos del pasado como acabar con la pobreza, la desigualdad, el hambre o proporcionar salud para todos. En este aspecto, Ecuador si dio un cambio trascendental desde la constitución de Monte Cristi (Asamblea Nacional del Ecuador, 2008) y ha logrado importantes transformaciones sociales siendo en la educación la gran esperanza de asentar otro modelo socio-económico.

Tal como se ha indicado, las competencias permiten evaluar el crecimiento personal a lo largo del proceso educativo, sin embargo es necesario diseñar nuevas fórmulas que puedan darnos mayor capacidad de comprender si realmente se están adquiriendo valores de responsabilidad social y compromiso humano. Si bien no depende solo del trabajo desde la Universidad en la Educación Superior, si es en esta etapa cuando debe hacerse un mayor examen dado que en manos de los egresados estará gestionar e impulsar el legado de avances sociales conseguidos (Cardona Arenas, Morales Marín, Cárdenas Aguirre \& Ramírez Castañeda, 2015). Las tecnologías abren espacios a conocimientos no mediados por un docente, si bien se puede relativizar su trascendencia, pues el caso de espacios pluriculturales como el de Ecuador u otros países de la región latinoamericana, hacen que aulas en las que se comparten diferentes cosmovisiones constituyan también un referente de cómo el docente es capaz de mediar entre conocimientos para construir un saber colectivo que bebe de diferentes fuentes. Al respecto, la tecnología es considerada una herramienta más, y como tal puesto que su acceso cada vez deviene más al alcance de cualquiera es menester formar a formadores, pero no solamente, sino a toda la comunidad para participar en el uso y debate de alcances y delimitaciones para contribuir a la educación. 
A modo de conclusión, resulta indispensable formarse continuamente como docentes para formar a las nuevas generaciones que a su vez interactúan presencial y virtualmente en diferentes planos de tiempo espacio, dando lugar un ciclo continuo que permita asentar los avances conseguidos en Ecuador. El modelo de competencias permite poner nombre a una serie de maneras de comprender, saber ser, saber hacer, saber conocer y saber convivir (Aguerrondo, 2009) que independiente de la herramienta o espacio de formación, permite independientemente de la cultura poner una base común. En este aspecto, el aprendizaje significativo supone el marco pertinente para enlazar prácticas culturales con un fondo común de valores a compartir a lo largo del proceso. No sabemos si las tecnologías serán perpetuas, sin embargo el bien común impulsado por formar en la responsabilidad social si brinda mayores garantías para avanzar hacia un desarrollo humanista en armonía con el medioambiente valorando la biodiversidad como riqueza.

\section{REFERENCIAS BIBLIOGRÁFICAS}

Adams Becker, S., Cummins, M., Davis, A., Freeman, A., Hall Giesinger, C., and Ananthanarayanan, V. (2017). NMC Horizon Report: 2017 Higher Education Edition. Austin, Texas: The New Media Consortium. Disponible en: https://goo.gl/3yqPYp

Adell, J. \& Castañeda, L. (2012). Tecnologías emergentes, ipedagogías emergentes? En J. Hernández, M. Pennesi, D. Sobrino \& A. Vázquez (Eds.), Tendencias emergentes en Educación con TIC (pp. 13-32). Barcelona: Espiral, Educación y Tecnología. Disponible en: http://goo.gl/nhbKVk

Aguerrondo, I. (2009) "Conocimiento complejo y competencias educativas", Working papers on curriculum issues, núm. 8, IBE/UNESCO. Disponible en: www.ibe.unesco.org/en/services/publications/ibe-working-papers.htm

Asamblea Nacional Constituyente (2008). Constitución de la República de Ecuador. Quito: Registro oficial.

Asamblea Nacional República del Ecuador [ANRE] (2010). Ley Orgánica de Educación Superior. Suplemento del Registro Oficial No. 298, de 12 de octubre de 2010. Quito: Registro Oficial de Ecuador.

Asamblea Nacional República del Ecuador [ANRE] (2016). Código orgánico de la economía social de los conocimientos, creatividad e innovación. Año IV, no 899, Quito: Registro Oficial Ecuador. Disponible en:: https://goo.gl/g1Lptn [Accedido Mayo 2017].

Benavides, F. \& Pedró, F. (2007). Políticas educativas sobre nuevas tecnologías en los países Iberoamericanos. Revista Iberoamericana de Educación, 45, 19-69. Disponible en: https://goo.gl/QXKJSs

C21 Canada (Canadians for 21st Century Learning and Innovation). (2012). Shifting minds: A 21 st century vision of public education for Canada. Disponible en: www.c21canada.org/wp-content/uploads/2012/11/Shifting-Minds- Revised.pdf 
Cardona Arenas, P., Morales Marín, J., Cárdenas Aguirre, D., \& Ramírez Castañeda, L. (2015). Papel de la educación superior en los procesos de formación en emprendimiento. Sinapsis, 7(7), 61-69. Disponible en: https://goo.gl/hpuFbo

Claro, M. (2010). La Incorporación de Tecnologías Digitales en Educación. Modelos de identificación de buenas prácticas. Santiago de Chile, CEPAL - Colección documentos de proyectos. Disponible en:: https://goo.gl/5vhQLd

Centro Europeo para el Desarrollo de la Formación Profesional (2014). Terminology of European education and training policy (Second Edition) - A selection of 130 key terms. Luxembourg: Publications Office. Disponible en: www.cedefop.europa.eu/EN/Files/4117_en.pdf

Consejo de Evaluación, Acreditación y Aseguramiento de la calidad de la Educación Superior [CEAACES] (2015). Adaptación del Modelo de Evaluación Institucional de Universidades y Escuelas Politécnicas 2013 al Proceso de Evaluación, Acreditación y Recategorización de Universidades y Escuelas Politécnicas. Quito: Dirección de Acreditación de Universidades y Escuelas Politécnicas.

Dede, C. (2014). The role of digital technologies in deeper learning. Students at the Center: Deeper Learning Research Series. Boston, MA: Jobs for the Future.

Delgadillo Franco, O. \& Islas Torres, C.; (2016). La inclusión de TIC por estudiantes universitarios: una mirada desde el conectivismo. Apertura, 8, 116-129.

doi: http://dx.doi.org/10.18381/Ap.v8n2.845

Drake, S.M. (2014). Designing across the curriculum for "sustainable well-being": A 21st century approach. En F. Deer, T. Falkenberg, B. McMillan, \& L. Sims (Eds.), Sustainable well-being: Concepts, issues, and educational practice (pp. 57-76). Winnipeg, MB: Education for Sustainable Well-Being (ESWB) Press.

Dumont, H., Istance, D., \& Benavides, F. (Eds.). (2010). The nature of learning: Using research to inspire practice. Paris: Educational Research and Innovation, OECD Publishing. Disponible en: http://dx.doi. org/10.1787/9789264086487-en

Fullan, M., \& Langworthy, M. (2014). A rich seam: How new pedagogies find deep learning. London: Pearson.

López, S; Veit, E A; Solano Araujo, I; (2014). La formulación de preguntas en el aula de clase: un a evidencia de aprendizaje significativo crítico. Ciência \& Educação, 20, 117-132. Disponible en: https://goo.gl/s8YrYa [Accedido Mayo 2017].

Martí Noguera, J. J.; Arango, O. E. y Moncayo, J. E. (2015), Experiencias iberoamericanas en responsabilidad social universitaria, Medellín, Universidad Luis Amigó. Disponible en: http://goo.gl/2ASRwT [Accedido en Marzo 2017].

Melo, D. F.; Silva Chávez, J. A.; Indacochea Mendoza, L. R. y Núñez Campaña, J. H. (2017). Tecnologías en la Educación Superior. Políticas Públicas y Apropiación Social en su Implementación. Revista Digital de Investigación y Docencia Universitaria.

Ministerio de Educación (2012). Estándares de Calidad Educativa. Aprendizaje, Gestión Escolar, Desempeño Profesional e Infraestructura. Quito, Autor.

Montero Pereda, J. (2014). Alfabetización digital de los estudiantes egresados del VII ciclo de educación básica regular en Chimbote, Ancash, Perú. In Crescendo, 4(1), 53-60.

Disponible en: <https://goo.gl/iYtBPg > . Fecha de acceso: 20 may. 2017.

Doi: http://dx.doi.org/10.21895/in cres.v4i1.20 
New Media Consortium (2013). Perspectivas Tecnologías. Educación Superior en América Latina 2013-2018. Un Análisis Regional del Informe Horizon del NMC. Horizon Project. Austin, Texas: The New Media Consortium. Disponible en: https://goo.gl/Uy62eu

Nizama Reyes, M. (2016). Caracterización y evolución de la tecnología educativa en el Perú. In Crescendo, 7(1), 71-76. Disponible en: < https://goo.gl/CpcAZb >

Doi: http://dx.doi.org/10.21895/in cres.v7i1.1166

Organization for Economic Co-operation and Development (2014). PISA 2012 results: Creative problem solving (volume V): Students' skills in tackling real-life problems. Paris: OECD Publishing. Disponible en: http://dx.doi.org/10.1787/9789264208070-en

Organization Economic Cooperation Development (2015), Students, Computers and Learning: Making the Connection, OECD Publishing, Paris.

Doi: http://dx.doi.org/10.1787/9789264239555-en

Organization Economic Cooperation Development (2016), Education at a Glance 2016: OECD Indicators, Paris: OECD Publishing. http://dx.doi.org/10.187/eag-2016-en [Accedido Mayo 2017].

Olaya, A. y Ramírez, J; (2015). Tras las huellas del aprendizaje significativo, lo alternativo y la innovación en el saber y la práctica pedagógica. Revista Científica Guillermo de Ockham, 13() 117-125. Disponible en: https://goo.gl/UFQISO [Accedido Mayo 2017]

Rafael, Á. (2015). Sentido y orientación de la política académica universitaria en un contexto global. In Crescendo, 6(2), 153-168. Disponible en: <https://goo.gl/mYcX42> Doi: http://dx.doi.org/10.21895/in cres.v6i2.1076

Rychen, D.S. (2003). Key competencies: Meeting important challenges in life. In D.S. Rychen \& L.H. Salganik (Eds.), Key competencies for a successful life and a well-functioning society (pp. 63-107). Göttingen: Hogrefe \& Huber Publishers.

Secretaría Nacional de Planificación y desarrollo (2013). Plan Nacional de Desarrollo / Plan Nacional para el Buen Vivir 2013-2017. Quito: SENPLADES. Disponible en: https://goo.gl/V5wXfp [Accedido Mayo 2017].

Sobrino Morrás, A. (2014). Aportaciones del conectivismo como modelo pedagógico postconstructivista. Propuesta Educativa, () 39-48. Disponible en: <https://goo.gl/Jxe02P> [Accedido Mayo 2017]

Tamayo, O. E., Zona, R., \& Loaiza, Y. E. (2015). El pensamiento crítico en la educación. Algunas categorías centrales en su estudio. Revista Latinoamericana de Estudios Educativos, 11(2), 111-133. Disponible en: https://goo.gl/eQ9dHl

Trilling, B., \& Fadel, C. (2009). 21st century skills: Learning for life in our times. San Francisco: Jossey-Bass.

World Economic Forum (2016). The Future of Jobs Employment, Skills and Workforce Strategy for the Fourth Industrial Revolution. Global challenge inside report. Geneva: WEF. Disponible en: http://www3.weforum.org/docs/WEF_Future_of_Jobs.pdf [Accedido Mayo 2017].

Zapata-Ros, M. (2015). Teorías y modelos sobre el aprendizaje en entornos conectados y ubicuos. Bases para un nuevo modelo teórico a partir de una visión crítica del "conectivismo". Teoría de la Educación. Educación y Cultura en la Sociedad de la Información, 16(1), 69-102. Disponible en: https://goo.gl/Rfaljz 